\title{
Evaluación de la implantación de la metodología Design Thinking en una asignatura de proyectos
}

\section{Enrique Costa-Montenegro ${ }^{\mathrm{a}}$, Francisco Díaz-Otero ${ }^{\mathrm{b}}$, Manuel Caeiro-Rodríguez ${ }^{\mathrm{a}}$, Iñigo} Cuiñas-Gómez ${ }^{b}$, Perfecto Mariño-Espiñeira ${ }^{c}$ y Manuel Fernández-Iglesias ${ }^{a}$

${ }^{a}$ Dept. Enxeñaría Telemática, Universidade de Vigo (kike@gti.uvigo.es, manuel.caeiro@det.uvigo.es, manuel.fernandez@det.uvigo.es), ${ }^{b}$ Dpto. Teoría do Sinal e Comunicacións, Universidade de Vigo (fjdiaz@com.uvigo.es, inhigo@uvigo.es) y ${ }^{c}$ Dpto. Tecnoloxía Electrónica, Universidade de Vigo (pmarino@uvigo.es)

\begin{abstract}
This paper discusses the experience of introducing the Design Thinking methodology in course Project Lab (LPRO) in academic year 2015/2016. $L P R O$ is a course in the syllabus of the degree in Telecommunication Technologies Engineering taught at University of Vigo, Spain. First, the target course is introduced, as it can be considered to be a unique course in a similar way to the Design Thinking methodology applied. Then, we discuss how Design Thinking was approached in three from the nineteen projects carried out during the mentioned academic year, paying special attention to the restrictions related to the time teachers could devote to interact with students in actual lectures. Finally, some results and concluding remarks are offered.
\end{abstract}

Keywords: Design Thinking, Project laboratory, Project-based learning, assessment.

\footnotetext{
Resumen

En esta comunicación relataremos la experiencia desarrollada durante el curso 2015/2016 al implantar la metodología Design Thinking en la asignatura Laboratorio de Proyectos (LPRO) impartida en el Grado en Ingeniería de Tecnologías de Telecomunicación de la Universidad de Vigo. Para ello presentaremos la asignatura, dado que se trata de una asignatura singular dentro del plan de estudios de esta titulación de grado, al igual que la metodología Design Thinking. Posteriormente explicaremos cómo hemos introducido dicha metodología en tres de los diecinueve proyectos llevados a cabo en la asignatura, sobre todo teniendo en cuenta las restricciones de horas presenciales dedicadas al trabajo en clase con el profesor. Finalmente presentaremos los resultados y conclusiones extraidos de esta experiencia.
}

Palabras clave: Design Thinking, laboratorio de proyectos, aprendizaje basado en proyectos, evaluación. 


\section{Introducción}

Dentro del Grado en Ingeniería de Tecnologías de Telecomunicación impartido en la Universidad de Vigo, en el cuarto curso, durante el segundo cuatrimestre se imparte la asignatura Laboratorio de Proyectos (LPRO) (Guía docente de la asignatura Laboratorio de Proyectos, Curso 2015/2016). Se trata de una asignatura obligatoria de 12 ECTS, que por su propia entidad tiene un carácter singular dentro de la titulación. Los proyectos deben ser interdisciplinares, en dos sentidos: (i) deben ser abordados por un equipo de estudiantes, normalmente entre 5 ó 6 , que tienen que representar por lo menos dos de las cuatro Menciones del Grado en Ingeniería de Tecnologías de Telecomunicación; (ii) los equipos son supervisados por dos profesores de departamentos diferentes para enriquecer y facilitar las sinergias entre diferentes áreas de trabajo.

Los alumnos de esta asignatura tienen 14 horas de clase presenciales con los coordinadores de la asignatura, básicamente centradas en actividades introductorias, seguimiento de los proyectos y exposición final. Estos proyectos desarrollados por los diferentes equipos serán defendidos al final de curso como parte del proceso de evaluación de la materia. Además tienen 7 horas de clase presenciales con los profesores supervisores del proyecto. Durante estas 7 horas se debe presentar la metodología a seguir y realizar el seguimiento del proyecto. El resto de horas, hasta cumplir los 12 ECTS de la asignatura, corresponden a trabajo autónomo del estudiante.

Cabe destacar que como resultado del aprendizaje de la asignatura LPRO no se valora el proyecto en sí o su grado de finalización, sino básicamente las capacidades de trabajar en grupo en desarrollo de proyectos. Más concretamente, los resultados de aprendizaje esperados al concluir la asignatura son: "Aprender a trabajar en grupo en un proyecto a medio plazo", "Planificar la temporalidad de un proyecto en grupo", "Integrar las habilidades propias en un grupo multidisciplinar" y "Mantener una actitud dinámica y potenciar el espíritu de superación".

La evaluación de la asignatura se divide en dos partes. Un 35\% corresponde a la evaluación de las presentaciones y exposiciones de los proyectos, realizada por los coordinadores de la asignatura. El $65 \%$ restante se reparte entre los profesores supervisores del proyecto, que evaluarán el seguimiento adecuado del proyecto, y una evaluación entre pares, donde los propios compañeros dentro del grupo del proyecto evaluarán el trabajo del estudiante.

La metodología Design Thinking (DT) (Johansson-Sköldberg, 2013; Owen, 2007), conocida también como Pensamiento para el Diseño, es una metodología para el desarrollo de proyectos que se basa en aplicar el enfoque y las técnicas habituales del mundo del diseño y la publicidad a disciplinas como la ingeniería, las ciencias empresariales o las ciencias sociales. Esta aproximación al desarrollo se basa en un enfoque novedoso organizado en una serie de etapas, identificadas en la mayoría de los casos como empatía, definición, generación de ideas, prototipado y experimentación. El Design Thinking fue desarrollado en el Institute of Design at Standford como una aproximación novedosa que facilitara la innovación frente a otras técnicas tradicionales. De hecho, además de convertirse en una metodología académica orientada al aprendizaje basado en proyectos, 
está siendo aplicada en numerosos proyectos reales en todo tipo de sectores, desde el marketing a la ingeniería. Podemos decir que con esta metodología se facilita de manera clara el desarrollo de soluciones innovadoras que a su vez están conectadas con las necesidades reales de los usuarios, mediante un enfoque centrado en las personas y no en casos de uso abstractos, basado en la realización de pruebas iterativas con prototipos de cada vez mayor complejidad.

En el curso 2015/2016 se han matriculado estudiantes para crear diecinueve grupos en la asignatura LPRO. Como experiencia innovadora en este curso hemos decidido que tres de estos grupos sigan la metodología Design Thinking. En esta comunicación explicaremos cómo hemos aplicado esta metodología en estos tres grupos. Para ello, la segunda sección de este documento describe los objetivos que nos marcamos en esta propuesta. La tercera sección está constituida por la descripción de las actividades realizadas en cada hora en cada grupo adaptándolas a esta metodología. En la cuarta sección evaluamos los resultados, principalmente de forma subjetiva, dado de que todavía no disponemos de resultados objetivos. Finalmente, la quinta sección recoge las conclusiones de la misma.

\section{Objetivos}

Uno de los objetivos principales de esta propuesta es adaptar la metodología Design Thinking para poder ser utilizada dentro de la asignatura LPRO por parte de los profesores supervisores de los proyectos. Hay que tener en cuenta que dichos profesores cuentan con muy pocas horas presenciales con los estudiantes, en concreto 7, por lo cual la metodología no se podrá implementar en toda su extensión. Como ejemplo, los cursos de Design Thinking de referencia tienen una duración de alrededor de 25 horas (Cramer \& Babwahsingh, 2015; Schindall \& Kotelly, 2015).

Cabe destacar que la mayoría de los estudios diferencian cinco fases principales en la metodología Design Thinking: (i) descubrimiento/empatía, (ii) interpretación del problema, (iii) ideación de la solución, (iv) prototipado y (v) pruebas. Estas fases no se suceden secuencialmente en el tiempo, sino que resultados de fases posteriores pueden llevar a cambios en fases previas. Veremos en la próxima sección cómo las aplicamos en nuestro caso.

Otro de los principales objetivos de esta propuesta es evaluar el grado de aceptación por parte del profesorado y del alumnado de esta metodología. El profesorado podrá mostrar su opinión sobre el alcance de los resultados de aprendizaje usando esta metodología, sobre todo en comparación con cursos anteriores. El alumnado, al no poder realizar esta comparación, mostrará su opinión sobre el desarrollo de la asignatura con dicha metodología.

\section{Desarrollo de la innovación}

En esta sección se describe cómo se ha adaptado la metodología Design Thinking para encajarla dentro de las 7 horas de clase presenciales que tienen los estudiantes con los profesores supervisores del proyecto. Vamos a dividir esta sección en subsecciones de 
acuerdo a cada una de las horas con el profesor, indicando las actividades realizadas durante esa hora en relación con la metodología, así como las actividades previas necesarias para la clase y las que deberán de hacer en las horas de trabajo autónomo para la siguiente clase.

\subsection{Primera hora: introducción y fase de descubrimiento/empatía}

Como requisito previo a la primera reunión se solicita a los estudiantes que vean un vídeo introductorio sobre la metodología a seguir, Design Thinking (Stanford Design Thinking Virtual Crash Course). Al principio de la clase, por parte de los profesores supervisores, se comenta el vídeo y se resuelven las diferentes cuestiones sobre la metodología que puedan aparecer.

A continuación se les plantea el proyecto que deben resolver los alumnos. Es muy importante que la especificación del proyecto sea lo más abierta posible, dado que son los alumnos los que deberán descubrir los requisitos y necesidades a cubrir gracias a la primera fase de esta metodología, la de descubrimiento/empatía. Si el proyecto ya se plantea cerrado desde el principio, esta primera fase no tendría mucho sentido.

Lo importante es analizar y comprender el desafío dado gracias a los posibles usuarios del hipotético producto que salga del proyecto y sus necesidades, no de las ideas preconcebidas de los propios estudiantes miembros del grupo. Para ello los estudiantes deberán salir de su habitual entorno de trabajo, las clases, al mundo real y realizar entrevistas con los posibles futuros usuarios de su proyecto para saber cómo les gustaría a estos que fuese y qué características debería tener.

Se requiere por tanto a los estudiantes que para la siguiente reunión hayan realizado entrevistas a posibles usuarios del futuro producto y se les especifica cómo hacerlas. Básicamente, es importante que el estudiante muestre una actitud afable e interesada hacia el entrevistado. Hay que tener en cuenta que en esta asignatura se realizan proyectos tecnológicos, normalmente innovadores, y que la persona entrevistada no suele posee este tipo de conocimientos. Por lo tanto, es necesario mostrar una actitud interesada hacia el usuario, no hacerle sentir intimidado dado que el entrevistador es un futuro graduado en telecomunicación, sino que sus respuestas son importantes dado que son las que van a definir los requisitos del proyecto. De la misma forma, no es conveniente plantear unas preguntas prefijadas, sino que es más recomendable dejar fluir la conversación y usar preguntas abiertas. Es decir, tratar de evitar las preguntas con respuesta sí o no, que cierran dicha respuesta del entrevistado sobre un tema concreto, sino hacer preguntas en las que el entrevistado tenga que expresar su opinión o selección sobre un tema y argumentarla.

\subsection{Segunda hora: interpretación del problema}

Como inicio de esta hora de clase se comentan las experiencias con las entrevistas, parte final de la fase de descubrimiento. Nuestros estudiantes en este curso han llegado con una media de 5 o 6 entrevistas cada uno, lo que nos dio un total de 25 o 30 entrevistas, dependiendo del grupo. 
Cabe destacar cómo estas entrevistas han hecho que nuestros alumnos se hayan dado cuenta de la importancia de obtener información directamente del usuario final del hipotético proyecto, descartando ideas preconcebidas sobre necesidades para dicho proyecto, así como posibles soluciones que inevitablemente ya se habían planteado.

En esta segunda fase, la de interpretación del problema, se pretende sintetizar los hallazgos obtenidos gracias a las entrevistas utilizando diferentes herramientas visuales, como pueden ser el mapa de empatía, el mapa de afinidad, la matriz de relaciones o el mapa conceptual. El objetivo es encontrar percepciones o intuiciones sobre lo aprendido en la fase anterior, y finalizar formulando el llamado Punto de Vista (POV, Point of View) que sirva como definición del problema a resolver.

Dada la restricción temporal anteriormente comentada, en esta hora se realizará un único mapa de empatía, si bien es cierto que se les comenta a los alumnos que en caso de identificar posible futuros usuarios claramente diferenciados, debería realizarse uno por cada uno de ellos.

Para este mapa de empatía usaremos el esquema presentado en la Figura 1, donde podemos ver la cabeza del usuario en el medio de la figura, al que le hemos asignado un nombre para hacerlo más personalizado. Este usuario estará dibujado en el centro de una pizarra, y se dividirá ésta en cuatro partes a cada lado del usuario relacionadas con la experiencia del usuario cuando use el supuesto producto:

- ¿Qué piensa o siente?: qué le mueve, cuáles son sus preocupaciones, qué le importa (lo que dice y lo que creemos entender), cuáles son sus expectativas...

- ¿Qué escucha?: lo que oye en el entorno, qué sonidos, qué cosas le dicen, quién se lo dice, a través de qué medio se lo dicen...

- ¿Qué dice y hace?: comportamiento, qué le importa, con quién habla, si dice lo mismo que creemos que piensa...

- ¿Qué ve?: su entorno, sus amigos, sus problemas...

Cada una de estas zonas se rellenará con numerosos post-it con información obtenida de las diferentes entrevistas realizadas a los diferentes usuarios. En la Figura 2 podemos ver una imagen real de un mapa de empatía de un grupo de la asignatura LPRO.

\subsection{Tercera hora: interpretación del problema, continuación}

Una vez construido el mapa de empatía, se procede a despegar los post-it de dicho mapa y colocarlos aleatoriamente en una mesa.

Pasamos a la siguiente fase, el mapa del pensamiento (Mind Mapping), posiblemente una de las partes más difíciles de toda la metodología. La idea es agrupar el conocimiento obtenido del mapa de empatía en diferentes categorías. Dichas categorías no están predefinidas, sino que deben surgir de la agrupación que consideren adecuada los miembros del grupo. Este mapa suele ser muy cambiante durante su desarrollo. Cabe la posibilidad de que alguno de los post-it no encaje en ninguna categoría, en tal caso se deja sin agrupar. Una vez finalizado dicho mapa, habrá que nombrar cada una de las categorías que se han 
obtenido. En la Figura 3 podemos ver una imagen real de un mapa de conocimiento de un grupo de la asignatura LPRO.

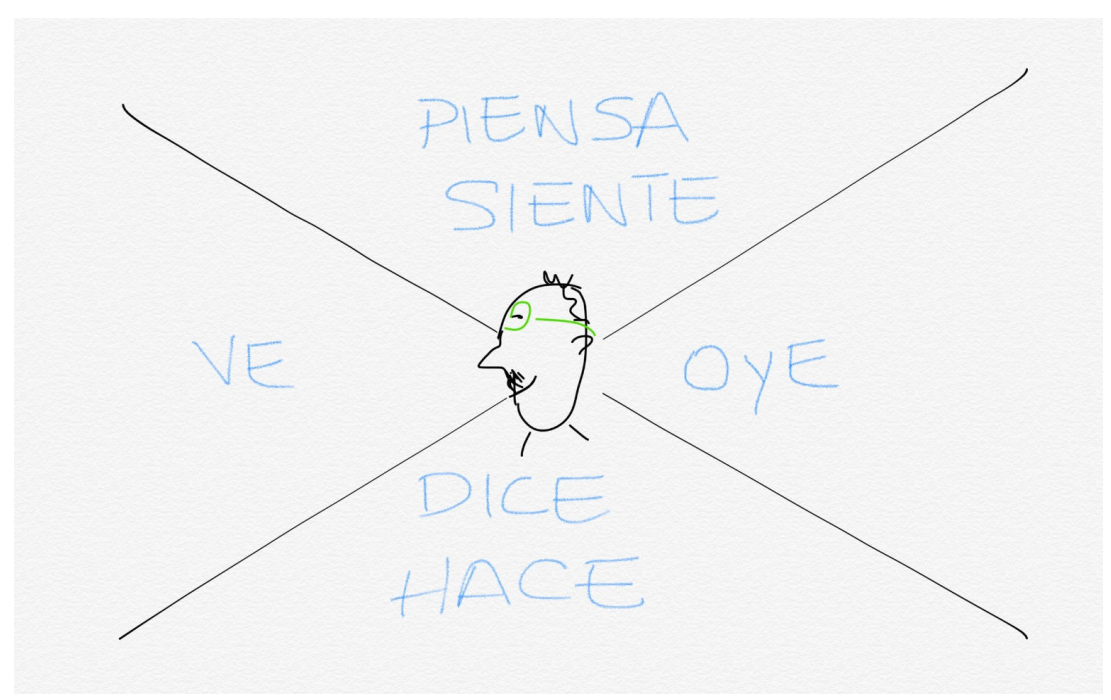

Fig. 1 Esquema del mapa de empatía

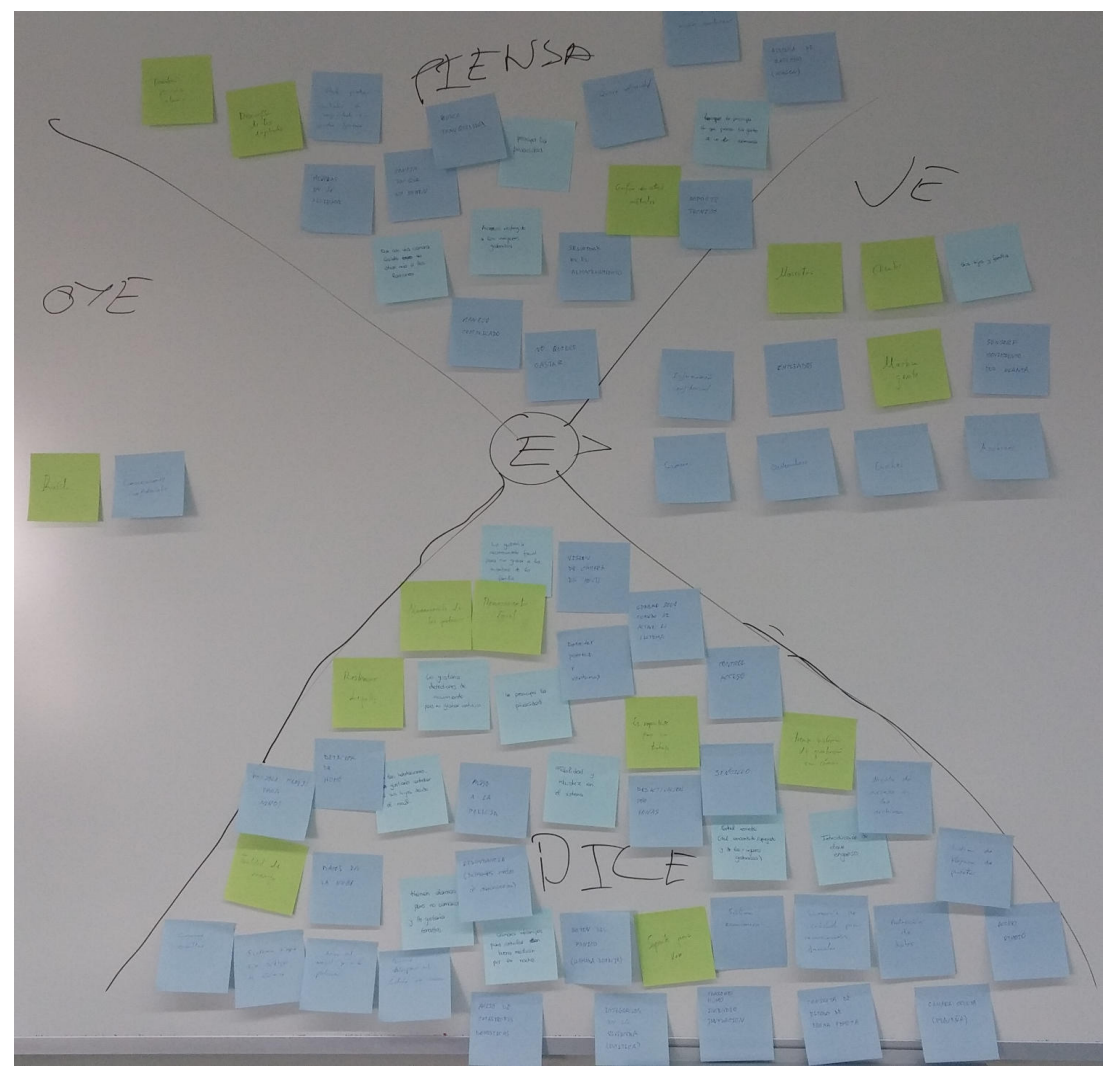

Fig. 2 Ejemplo de un mapa de empatía real en una clase de LPRO. 


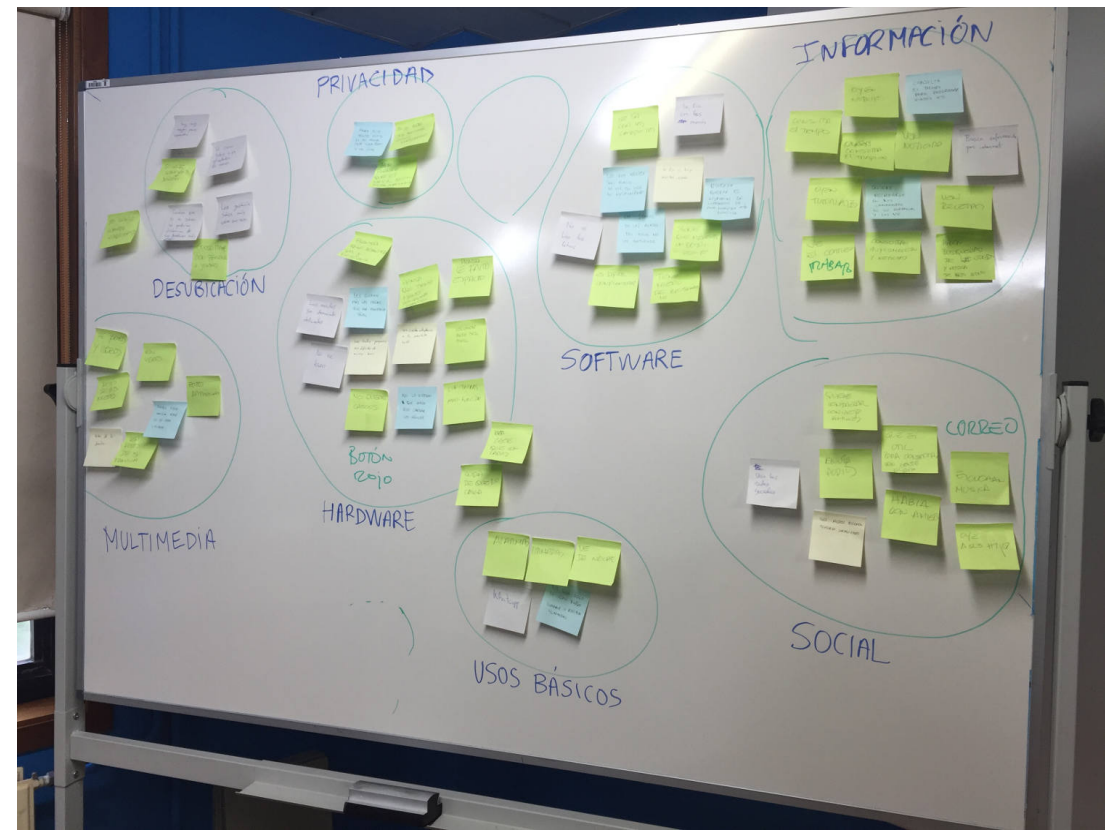

Fig. 3 Ejemplo de un mapa de conocimiento real en una clase de LPRO.

\subsection{Cuarta hora: ideación de la solución}

Al comienzo de la clase se revisa el Punto de Vista que han consensuado en las horas de trabajo autónomo, y los estudiantes reciben opinión de los profesores supervisores del proyecto.

Esta tercera fase, la ideación de la solución, tiene como objetivo que el grupo proponga una posible solución al proyecto definido por el Punto de Vista. Para ello es necesario definir preguntas basadas en dicho Punto de Vista. Las respuesta a dichas preguntas se suelen contestar mediante propuestas surgidas de técnicas de brain-storming (lluvia de ideas), siempre buscando diferentes puntos de enfoque, positivismo en las propuestas, y $\sin$ descartar ningún tipo de idea. Finalmente será necesario decidir qué conjunto de ideas propuestas se implementarán (cardsorting), para ello se pueden utilizar técnicas de votación, comparación, etc.

Durante esta hora se realiza un brain-storming donde los diferentes miembros del grupo deben aportar ideas para solucionar el proyecto, o parte de éste, definido por el Punto de Vista. Es importante inculcarle a los estudiantes que en este punto no existen malas ideas, se pueden poner ideas descabelladas, dado que más adelante habrá una fase para la elección de las ideas a resolver. También esta fase debe ser positiva, no se permite la descalificación de ideas de otros estudiantes, por ejemplo con frases empezando por "pero...", sino que hay que construir a partir de las ideas de los compañeros. Se pretende que este proceso sea visual, para lo cual se escriben las ideas en post-it y se pegan en la pizarra, además se hará de una en una, y el estudiante que la propone la dirá en alto para que los compañeros 
puedan construir sobre ella. Es importante acabar esta fase con el mayor número de ideas posibles.

\subsection{Quinta hora: ideación de la solución, continuación}

En esta segunda parte de la ideación de la solución se va a trabajar con todas las ideas resultantes del proceso de brain-storming. Es necesario decidir de todas esas ideas cuáles se van a intentar solucionar en el proyecto en curso. Para ello se utiliza un método de cardsorting. En particular, en nuestra asignatura LPRO hemos decidido utilizar un eje de coordenadas cartesianas, de forma que en la abscisa tenemos si la idea a ordenar "mola" y en la ordenada si es realizable. Los estudiantes van cogiendo los post-it con las ideas procedentes del brain-storming y las van colocando en este eje en diferentes posiciones según si "molan" o no y son realizables dentro de la asignatura o no. Es importante que la decisión sobre la colocación se tome por consenso entre todos los estudiantes. En la Figura 4 podemos ver una imagen real de un cardsorting utilizando el eje de coordenadas cartesianas propuesto en un grupo de la asignatura LPRO.

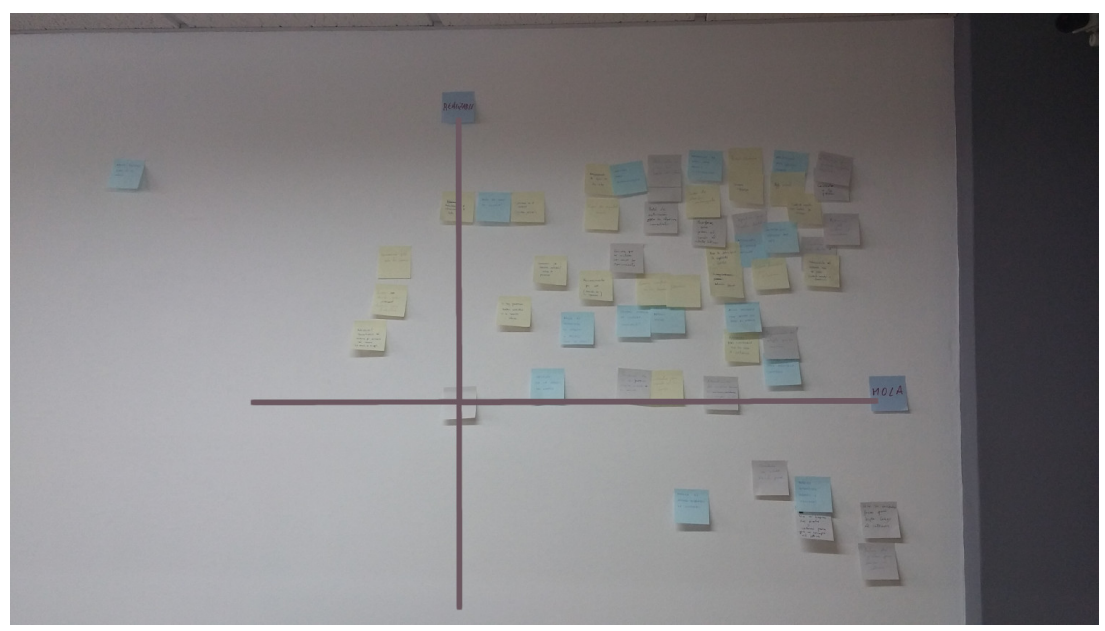

Fig. 4 Ejemplo de un cardsorting de ideas real en una clase de LPRO.

Lógicamente, las ideas que se van a utilizar para resolver el proyecto en cuestión son las que están en la zona "molan" y son realizables.

Finaliza la ideación con un boceto de la posible solución teniendo en cuenta las ideas elegidas en el cardsorting. Este fase se realiza de forma individual, así cada estudiante puede aportar al grupo una solución diferente. Se les pide que para la siguiente clase acuerden una solución común tomando como partida las diferentes soluciones individuales.

\subsection{Sexta y séptima hora: prototipado y test}

Estas fases son más habituales del desarrollo de un proyecto con una metodología clásica. Tienen como objetivo la creación de prototipos de baja fidelidad mediante el dibujo, maquetas o haciendo pequeñas manualidades, para poder presentar el prototipo ante un posible usuario final y que sea probado para obtener retroalimentación. Tiene también parte de retroalimentación, viendo la relación entre el Punto de Vista y la solución propuesta, 
pensar como iterar dentro de las fases previas de la metodología Design Thinking para llegar a una solución más precisa.

Al principio de estas fases se les pide a los estudiantes que presenten el primer prototipo resultante de consensuar los bocetos que han realizado cada uno de ellos individualmente. Han de defender este prototipo ante los profesores supervisores del proyecto y recibirán propuestas de éstos. Dado que esta asignatura se cursa en un grado en telecomunicación, normalmente el prototipo tendrá una parte hardware y otra software. No es necesario que la parte de prototipado hardware sea con componentes reales, se permite utilizar materiales del estilo plastilinas o cartón, dado que la idea es que se pueda ver cómo sería en la realidad. Lo mismo pasa con la parte software, dado que no es necesaria implementarla, sino que basta con explicar cómo funcionará y mostrar cómo sería el interfaz gráfico con el que interactuaría el usuario final.

Cabe destacar que la mayor carga de trabajo autónomo de los estudiantes está concentrada en esta parte, dado que es la parte de desarrollo real de la solución para el proyecto. Los profesores supervisores irán revisando los sucesivos prototipos y aportando sus opiniones para las mejoras de éstos.

De cara a la realización de los test, se destaca la importancia de realizar pruebas con los usuarios. Las bondades o deficiencias de los productos desarrollados no son determinadas por los propios estudiantes ni por los profesores, sino que deben ser los usuarios destinatarios de los productos los que valoren las mismas. En este sentido, se hace hincapié en que los estudiantes prueben los prototipos en la medida en que sea posible con los usuarios finales.

En cuanto a la realización de prototipos, se resalta la posibilidad de crear prototipos a distintos niveles de madurez y realismo. Se puede hacer un prototipo como un dibujo en una hoja de papel hasta un sistema electrónico con funcionalidades bastante cercanas a las del producto final. La finalidad del prototipo es poder hacer pruebas con los usuarios finales y obtener realimentación de los mismos. Lo realmente importante es poder obtener dicha realimentación lo antes posible, de forma que las ideas y propuestas realizadas en las distintas fases de la metodología puedan ser revisadas y actualizadas si ha lugar. A pesar de la mención al pensamiento (thinking) en la denominación de esta metodología, un punto clave del mismo se encuentra en la observación y consideración de los usuarios finales. Desde la empatía hasta las pruebas son sus puntos de vista los que deben ser tenidos en cuenta de cara al trabajo a realizar.

En estas dos últimas fases también se intenta promover que se hagan desarrollos incrementales, realizando los primeros prototipos lo antes posible, aunque en formas simples, para que puedan ser presentados a los usuarios y estos puedan proporcionar realimentación al proyecto. La idea que subyace a esta estrategia es que, en el desarrollo de productos, las primeras versiones suelen presentar problemas que es necesario corregir. Por tanto, antes que dedicar tiempo a la realización de análisis y diseños elaborados se requiere poner a prueba las ideas, recoger la realimentación de los usuarios y evolucionar los productos

2016, Universitat Politècnica de València

Congreso In-Red (2016) 


\section{Resultados}

En este curso 2015/2016 hemos utilizado por primera vez la metodología Design Thinking dentro de la asignatura de Laboratorio de Proyectos. Solo se ha aplicado esta metodología a tres de los diecinueve grupos matriculados en la asignatura. Todavía no poseemos resultados académicos objetivos para valorar esta experiencia, por ello vamos a intentar valorarla desde el punto de vista subjetivo de los profesores que la hemos impartido y autores de estos documentos. También haremos constar las opiniones que nos han hecho llegar nuestros alumnos sobre la metodología.

Desde el punto de vista del profesor, creemos que con esta metodología se cumple completamente con los objetivos de aprendizaje propuestos en la asignatura. Recordemos que la idea de esta asignatura es que los estudiantes aprendan a trabajar en grupo en el desarrollo de proyectos. Durante las clases con los profesores supervisores, éstos pueden apreciar perfectamente la cohesión de los grupos para el desarrollo del proyecto y la motivación que transmiten en las diferentes fases del desarrollo de la metodología, dado que se trata de una metodología muy dinámica. Una cuestión a destacar es que la metodología Design Thinking encaja perfectamente con las nuevas metodologías ágiles de desarrollo software. En éstas se consideran ciclos cortos de desarrollo de funcionalidades y la presencia de los usuarios finales durante las distintas fases de desarrollo es bienvenida.

La introducción en esta asignatura de la metodología Design Thinking ha supuesto una mejora significativa respecto a metodologías más tradicionales de Gestión de Proyectos aplicadas en la misma asignatura en cursos anteriores por los autores de este artículo. Esta mejora se evidencia tanto en el desarrollo y consecución de objetivos marcados, como en el grado de implicación de los estudiantes en los proyectos asignados. De manera cualitativa en la actitud de los estudiantes, en su nivel de satisfacción sobre los resultados obtenidos en cada proyecto y en la propia percepción subjetiva de dichos estudiantes respecto a la calidad trabajo realizado. Y, además, de manera objetiva, los productos y prototipos obtenidos en los grupos de trabajo responden de manera eficaz a necesidades reales de los clientes objetivo, lo cual incrementa el grado de satisfacción por el trabajo realizado.

Desde el punto de vista que nos transmiten los alumnos, si bien es cierto que al principio tienen un temor inicial dado que la primera fase, en las entrevistas, les obliga a interactuar con personas desconocidas, después no hacen más que mostrar su satisfacción con la metodología empleada. Se dan cuenta de lo oportuno que es empezar con la fase de descubrimiento y empatía para desterrar ideas preconcebidas que tenían desde el planteamiento del problema y trabajar solo con la información que han obtenido de los futuros usuarios finales. También muestran su agrado en las siguientes fases de interpretación del problema e ideación de la solución, dado que son fases muy visuales y dinámicas, muy alejadas del tipo de trabajo habitual que están acostumbrados a realizar en la mayoría de las asignaturas del grado. Es importante, en estas fases, consensuar las decisiones entre los estudiantes, de forma que se den cuenta de que su solución no es la única y existen diferentes formas de abordar el problema. Finalmente valoran muy positivamente la posibilidad de llegar a un prototipo inicial en muy poco tiempo, validado por todo el trabajo inicial realizado en la parte de descubrimiento.

(cc) EY-NC-ND 2016, Universitat Politècnica de València

Congreso In-Red (2016) 


\section{Conclusiones}

A lo largo de este artículo hemos explicado nuestra experiencia en la aplicación de la metodología Design Thinking en la asignatura Laboratorio de Proyectos del Grado en Ingeniería de Tecnologías de Telecomunicación de la Universidad de Vigo.

Hemos explicado que debido a las restricciones temporales ( 7 horas con los profesores supervisores del proyecto) es necesario reducir y concentrar las diferentes fases de esta metodología. A lo largo del artículo hemos detallado en qué empleamos las diferentes horas disponibles y las diferentes actividades que se realizan relacionadas con la metodología.

Finalmente, valoramos el uso de la metodología de forma subjetiva, debido a no disponer todavía de resultados debido a ser el primer curso en la que se aplica. Tanto los profesores supervisores como los estudiantes valoran positivamente esta metodología dado que les permite afrontar un proyecto de entidad pensando desde un principio en el usuario final y con la posibilidad de llegar a un prototipo inicial válido en poco tiempo.

\section{Agradecimientos}

Este trabajo ha sido realizado en el marco del proyecto "Development of Innovative AcadeMy ON the basis of DT teaching (DiamonDT)", referencia 2015-1-PL01-KA203016844, financiado por la Unión Europea, programa Erasmus+ key action 2. Su principal objetivo es proporcionar instrumentos para la introducción del Design Thinking como metodología educativa para el aprendizaje basado en problemas. Estos instrumentos incluyen materiales educativos, guías para la formación de docentes, cursos cortos de formación para docentes y estudiantes, y ejemplos de buenas prácticas. El consorcio DiamonDT está formado por la Universidad Politécnica de Lodz (Polonia), la Universidad de Ciencias y Tecnología de Bydgoszcz (Polonia), la Universidad de Tromso (Noruega), y la Universidade de Vigo.

\section{Referencias}

CRAMER, A. \& BABWAHSINGH, M.: "Design Thinking: A Creative Approach to Problem Solving and Creating Impact". Course Syllabus, PADM-GP 2145, New York University Wagner. $\quad<$ https://wagner.nyu.edu/files/syllabi/201306/PADMGP.2145.001.pdf $>$ [Consulta: 18 de marzo de 2016].

Guía docente de la asignatura Laboratorio de Proyectos, Curso 2015/2016. $<$ https://seix.uvigo.es/docnetnuevo/guia_docent/index.php?centre $=305 \&$ ensenyament $=\mathrm{V} 05 \mathrm{G} 300 \mathrm{~V} 01$ \&assignatura $=\mathrm{V} 05$ G300V01802\&idioma $=$ cast $>$ [Consulta: 3 de abril de 2016]

JOHANSSON-SKÖLDBERG, U., WOODILLA, J. y ÇETINKAYA, M. (2013). "Design Thinking: Past, Present and Possible Futures" en Creativity and Innovation Management, vol. 22, issue 2, p. 121-146.

(cc)) EY-NC-ND 2016, Universitat Politècnica de València

Congreso In-Red (2016) 
OWEN, C. (2007). "Design Thinking: Notes on its Nature and Use" en Design Research Quarterly, vol. 2, issue 1, p. 16-27.

SCHINDALL, J. \& KOTELLY, B.: Mastering Innovation and Design Thinking, Course Syllabus, Massachusetts Institute of Technology, 2015. $<$ http://web.mit.edu/professional/short-

programs/courses/mastering innovation_and_design thinking.html $>\quad[$ Consulta: $18 \mathrm{de}$ marzo de 2016].

"Stanford Design Thinking Virtual Crash Course". Youtube $<$ https://www.youtube.com/watch?v=-FzFk3E5nxM> [Consulta: 3 de abril de 2016] 